\title{
Income Distribution and Market Demand: The Case of Heterogeneous Preferences
}

\author{
Ibragimov M.
}

Tufetulov A.M.

Kazan Federal University, Institute of Management, Economics and Finance, Kazan, 420008, Russia

\section{Doi:10.5901/mjss.2014.v5n24p185}

\section{Abstract}

In this note, we obtain sufficient conditions under which changes in income inequality lead to an increase or decrease in the market demand elasticities in the case of heterogeneous preferences among the consumers. In this paper, we applied majorization theory to study dependence of market demand elasticity on the inequality in income distribution among the consumers. In this note, we extend the results obtained to the case where consumers' preferences are heterogeneous and the condition on equality of individual demand functions does not necessarily hold. This case is more realistic because consumers' preferences are affected by a variety of different factors.

Keywords: Income inequality, market demand, elasticity, heterogeneous preferences

In recent years, a number of studies have focused on modeling income inequality using majorization relation (see, e.g., Marshall and Olkin [6]) and applications of the latter concept to the problems in economics. The approach to the analysis of income inequality based on majorization which dates back to Lorenz [5] has been used, among others, by Atkinson [1], Dasgupta, Sen and Starrett [2], Shorrocks [8] and, more recently, Saposnik [7]. Using related concepts and methods, Lambert and Pfahler [4] presented an analysis of the effects of income (re-)distribution on the market demand for a good or service.

In [3], the authors applied majorization theory to study dependence of market demand elasticity on the inequality in income distribution among the consumers. However, in [3] it is assumed that consumers' preferences are the same for given prices on goods independently of their income levels. In this note, we extend the results obtained in [3] to the case where consumers' preferences are heterogeneous and the condition on equality of individual demand functions does not necessarily hold. This case is more realistic because consumers' preferences are affected by a variety of different factors.

Let there be $\mathrm{K}$ consumers and $\mathrm{M}$ goods in an economy. Denote by $\varphi_{m k}\left(P, I_{k}\right)$ the function of the kth consumer's demand on the mth good, by $\boldsymbol{I}=\left(I_{1}, \ldots, I_{K}\right)$ the vector of incomes of the consumers and by $\boldsymbol{P}=\left(p_{1}, \ldots, p_{M}\right)$ the vector of prices on goods.

Let $\Phi_{m}(\boldsymbol{P}, \boldsymbol{I})=\sum_{k=1}^{K} \varphi_{m k}\left(P, I_{k}\right)$ be the function of market (aggregate) demand on good $\mathrm{m}$ and let $e_{m}(\boldsymbol{I})=$ $\partial \log \Phi_{m}(\boldsymbol{P}, \boldsymbol{I}) / \partial \log p_{m}$ stand for its own-price elasticity. Denote by $S_{m k} \subset \mathbf{R}_{m+1}$ the domain of definition of the function $\varphi_{m k}\left(P, I_{k}\right)$ and by $S_{m}=\left\{(\boldsymbol{P}, \boldsymbol{I})=\left(\boldsymbol{P}, I_{1}, \ldots, I_{K}\right) \in \boldsymbol{R}^{M+K},\left(\boldsymbol{P}, I_{k}\right) \in S_{m k}, k=1, \ldots, K\right\}$ the domain of definition of the function $\Phi_{m}(\boldsymbol{P}, \boldsymbol{I}), m=1, \ldots, M$.

According to the idea going back to Lorenz [5] (see Marshall and Olkin [6]), a vector $\boldsymbol{I}^{(1)}=\left(I_{1}^{(1)}, \ldots, I_{K}^{(1)}\right.$ ) represents a more uniform distribution of the total income $Y$ among $K$ consumers than a vector $\boldsymbol{I}^{(2)}=\left(I_{1}^{(2)}, \ldots, I_{K}^{(2)}\right)$ if $\sum_{i=1}^{l} I_{[i]}^{(1)} \leq \sum_{i=1}^{l} I_{[i]}^{(2)}, \mathrm{I}=1, \ldots, \mathrm{K}-1$, and $\sum_{i=1}^{K} I_{[i]}^{(1)}=\sum_{i=1}^{K} I_{[i]}^{(2)}=Y$, where $I_{i}^{(j)}, \mathrm{j}=1,2$, are the income levels of the ith consumer and $I_{[1]}^{(j)} \geq I_{[2]}^{(j)} \geq \cdots \geq I_{[K]}^{(j)}$ denote the components of the vectors $I(j), \mathrm{j}=1,2$, in decreasing order (if the above conditions hold, it is said that the vector $\boldsymbol{I}^{(1)}=\left(I_{1}^{(1)}, \ldots, I_{K}^{(1)}\right)$ is majorized by $\boldsymbol{I}^{(2)}=\left(I_{1}^{(2)}, \ldots, I_{K}^{(2)}\right)$, written $\boldsymbol{I}^{(1)} \prec$ $\boldsymbol{I}^{(2)}$.

A function $\boldsymbol{f}(\boldsymbol{I})$ is called Schur-convex (resp., Schur-concave) in I if $\left(\boldsymbol{I}^{(1)} \prec \boldsymbol{I}^{(2)}\right) \Rightarrow\left(f\left(\boldsymbol{I}^{(1)}\right) \leq f\left(\boldsymbol{I}^{(2)}\right)\right)($ resp. $\left.\left(\boldsymbol{I}^{(1)} \prec \boldsymbol{I}^{(2)}\right) \Rightarrow\left(f\left(\boldsymbol{I}^{(1)}\right) \geq f\left(\boldsymbol{I}^{(2)}\right)\right)\right)$.

Theorem 1. (i) Let the individual demand functions $\varphi_{m k}\left(P, I_{k}\right)$ be twice continuously differentiable and let, for all $(\boldsymbol{P}, \boldsymbol{I}) \in S_{m}$ such that $I_{r} \leq I_{s}$, the following conditions hold:

$$
\frac{\partial \varphi_{m r}\left(P, I_{r}\right)}{\partial I_{r}} \leq \frac{\partial \varphi_{m r}\left(P, I_{S}\right)}{\partial I_{s}} \text {, (1) }
$$


$\frac{\partial^{2} \varphi_{m r}\left(P, I_{r}\right)}{\partial p_{m} \partial I_{r}} \leq \frac{\partial^{2} \varphi_{m r}\left(P, I_{s}\right)}{\partial p_{m} \partial I_{s}},(2)$

where pm is the price of the mth good in consideration. Then the absolute value of the elasticity |em(I)| is Schur concave in I on the set Sm. That is, the more non-uniform is the distribution of the total income among consumers in the economy, the smaller is the elasticity of the aggregate demand on the considered good by the absolute value.

(ii) If in conditions (1) and (2) the inequality sign $\leq$ is replaced by $\geq$, then the absolute value of the elasticity $\mid$ em(I)| is Schur-convex in I on Sm. That is, the more non-uniform is the distribution of the total income among the consumers, the larger is the elasticity of the aggregate demand on the considered good by the absolute value.

Proof. (i) Let $g_{m}(P, \boldsymbol{I})=\partial \Phi_{m}(\mathrm{P}, \boldsymbol{I}) / \partial \mathrm{p}_{m}=\sum_{k=1}^{K} \partial \phi_{m k}\left(\mathrm{P}, \boldsymbol{I}_{k}\right) / \partial \mathrm{p}_{m}$ be the deriva tive of the function of aggregate demand on the mth good with respect to its price. If conditions (1) and (2) are satisfied, then the following inequalities hold:

$\left(I_{r}-I_{S}\right)\left(\frac{\partial \Phi_{m}(P, I)}{\partial I_{r}}-\frac{\partial \Phi_{m}(P, I)}{\partial I_{S}}\right)=\left(I_{r}-I_{S}\right)\left(\frac{\partial \sum_{k=1}^{K} \phi_{m k}\left(P, I_{k}\right)}{\partial I_{r}}-\frac{\partial \sum_{k=1}^{K} \phi_{m k}\left(P, I_{k}\right)}{\partial I_{S}}\right)=\left(I_{r}-I_{S}\right)\left(\frac{\partial \Phi_{m r}\left(P, I_{r}\right)}{\partial I_{r}}-\frac{\partial \Phi_{m s}\left(P, I_{S}\right)}{\partial I_{S}}\right)$

and

$\left(I_{r}-I_{s}\right)\left(\frac{\partial g_{m}(P, \boldsymbol{I})}{\partial I_{r}}-\frac{\partial g_{m}(P, \boldsymbol{I})}{\partial I_{s}}\right)=\left(I_{r}-I_{S}\right)\left(\frac{\partial^{2} \Phi_{m r}(P, \boldsymbol{I})}{\partial p_{m} \partial I_{r}}-\frac{\partial^{2} \Phi_{m s}(P, \boldsymbol{I})}{\partial p_{m} \partial I_{s}}\right) \geq 0$

In addition, from the definition of the functions $\Phi_{m}(P, \boldsymbol{I})$ and $g_{m}(P, \boldsymbol{I})$ it follows that they are symmetric on the set Sm, that is,

$\Phi_{m}\left(P, I_{1}^{(1)}, \ldots I_{K}^{(1)}\right)=\Phi_{m}\left(P, I_{\pi(1)}^{(1)}, \ldots I_{\pi(K)}^{(1)}\right)$

$g_{m}\left(P, I_{1}^{(1)}, \ldots I_{K}^{(1)}\right)=g_{m}\left(P, I_{\pi(1)}^{(1)}, \ldots I_{\pi(K)}^{(1)}\right)$

for all permutations $\pi:\{1, \ldots, K\} \rightarrow\{1, \ldots K\}$ of the set $\{1, \ldots K\}$

Consequently, according to Theorem 3.A.4 in [6], the functions $\Phi_{m}(P, \boldsymbol{I})$ and $g_{m}(P, \boldsymbol{I})$ are Schur-convex in $\boldsymbol{I}$, that is, $\boldsymbol{I}^{(1)} \prec \boldsymbol{I}^{(2)}$ implies $\Phi_{m}\left(P, \boldsymbol{I}^{(1)}\right) \leq \Phi_{m}\left(P, \boldsymbol{I}^{(2)}\right)$ and $g_{m}\left(P, \boldsymbol{I}^{(1)}\right) \leq g_{m}\left(P, \boldsymbol{I}^{(2)}\right)$.

Since the function $g_{m}(P, \boldsymbol{I})$ is non-positive, from $\boldsymbol{I}^{(1)} \prec \boldsymbol{I}^{(2)}$ it thus follows that

$\frac{g_{m}\left(P, \boldsymbol{I}^{(1)}\right)}{\Phi_{m}\left(P, \boldsymbol{I}^{(1)}\right)} \leq \frac{g_{m}\left(P, \boldsymbol{I}^{(2)}\right)}{\Phi_{m}\left(P, \boldsymbol{I}^{(2)}\right)}$

or, equivalents

$e_{m}\left(\boldsymbol{I}^{(1)}\right)=\frac{\partial \Phi_{m}\left(P, \boldsymbol{I}^{(1)}\right)}{\partial p_{m}} \cdot \frac{p_{m}}{\Phi_{m}\left(P, \boldsymbol{I}^{(1)}\right)} \leq \frac{\partial \Phi_{m}\left(P, \boldsymbol{I}^{(2)}\right)}{\partial p_{m}} \cdot \frac{p_{m}}{\Phi_{m}\left(P, \boldsymbol{I}^{(2)}\right)}=e_{m}\left(\boldsymbol{I}^{(2)}\right)$

That is, $\boldsymbol{I}^{(1)} \prec \boldsymbol{I}^{(2)} i$ mplies $\left|e_{m}\left(\boldsymbol{I}^{(2)}\right)\right| \leq\left|e_{m}\left(\boldsymbol{I}^{(1)}\right)\right|$, as claimed.

(ii) If in conditions (1) and (2) the inequality sign $\leq$ is replaced by $\geq$, then the functions $\Phi_{m}(P, \boldsymbol{I})$ and $g_{m}(P, \boldsymbol{I})$ are Schur-concave in I, that is, $\boldsymbol{I}^{(1)} \prec \boldsymbol{I}^{(2)}$ implies $\Phi_{m}\left(P, \boldsymbol{I}^{(1)}\right) \geq \Phi_{m}\left(P, \boldsymbol{I}^{(2)}\right)$ and $g_{m}\left(P, \boldsymbol{I}^{(1)}\right) \geq g_{m}\left(P, \boldsymbol{I}^{(2)}\right)$ The rest of the arguments is completely similar to part (i).

Example 1. Suppose that the function of market demand for good $m$ has the CES form: $\Phi_{m}(P, \boldsymbol{I})=$ $\sum_{i=1}^{K} \phi\left(P, \alpha_{[i]}, I_{[i]}\right)$, where $I[1] \geq I[2] \geq \ldots \geq I_{[K]}, 1 \geq \alpha_{[1]} \geq \alpha_{[2]} \geq \ldots \geq \alpha_{[K]}>1 / 2, \phi(P, \alpha, I)=\psi(P, \alpha)$,$I and$

$\psi(P, \alpha)=,\frac{p_{m}^{-1 /(1-\alpha)}}{\sum_{j=1}^{M} p_{j}^{-\alpha /(1-\alpha)}}$

are the factors at the individual CES utility functions (that is, the consumers with a higher income I have a higher elasticity of substitution $-1 /(1-\alpha))$. We have

$$
\begin{aligned}
& \frac{\partial \phi\left(P, \alpha_{r}, I_{r}\right)}{\partial I_{r}}=\frac{1}{p_{m}\left(\sum_{j=1}^{M}\left(p_{j} / p_{m}\right)^{-\alpha_{r} /\left(1-\alpha_{r}\right)}\right)} \\
& \frac{\partial \phi\left(P, \alpha_{r}, I_{r}\right)}{\partial p_{m} \partial I_{r}}=\frac{\left(\alpha_{r}-\sum_{j=1}^{M}\left(p_{j} / p_{m}\right)^{-\alpha_{r} /\left(1-\alpha_{r}\right)}\right)}{p_{m}^{2}\left(1-\alpha_{r}\right)\left(\sum_{j=1}^{M}\left(p_{j} / p_{m}\right)^{-\alpha_{r} /\left(1-\alpha_{r}\right)}\right)}
\end{aligned}
$$

Simce the function $\left(\sum_{j=1}^{M}\left(p_{j} / p_{m}\right)^{-\alpha_{r} /\left(1-\alpha_{r}\right)}\right)^{-1}$ is increasing in $\alpha \in(0,1)$ for $p_{j} \geq p_{m}, j=1, \ldots, M, j \neq m$, we have that $\Phi_{m}(\mathrm{P}, \boldsymbol{I})$ satisfies conditions (1) if $p_{j} \geq p_{m}, j=1, \ldots, M, j \neq m$. Further, since the function $h(x)=$ $\alpha x^{2}-x$ is increasing in $\mathrm{x}$ for $x \geq 1 /(2 \alpha)$, we get that $\Phi_{m}(\mathrm{P}, \boldsymbol{I})$ satisfies conditions (2) if $p_{j} / p_{m} \geq \max _{i=1, \ldots, K}\left(\frac{M-1}{2 \alpha_{i}-1}\right)^{\left(1-\alpha_{i}\right) / \alpha_{i}}$ for $j=1, \ldots, M, j \neq m$. From part (i) of Theorem 1 we obtain that, in this domain, an increase in income inequality leads to a decrease in the absolute value of the market demand elasticity.

Similarly, in the above domain, the market demand function $\Phi_{m}(\mathrm{P}, \boldsymbol{I})=\sum_{i=1}^{K} \phi\left(P, \alpha_{(i)}, I_{[j]}\right)$, where $\alpha_{(1)} \leq \alpha_{(2)} \leq$ $\cdots \leq \alpha_{(K)}$ and $I_{[1]} \geq I_{[2]} \geq \cdots \geq I_{[K]}$ are ordered in the opposite ways, satisfies conditions (1) and (2) with the inequality signs $\leq$ replaced by $\geq$. From part (ii) of Theorem 1 we conclude that, in this case, an increase in income inequality leads to an increase in the absolute value of the market demand elasticity.

Example 2. Suppose that the function of market demand for good $m$ has the form 


$$
\Phi_{m}(p, I)=\sum_{i=1}^{K} \phi\left(p, \alpha_{i}, \beta_{i}, I_{[i]}\right) \text {, where } \phi(p, \alpha, \beta, I)=\frac{\alpha I}{\left(I+\beta_{p}\right)} \text { is a typical function on goods of first }
$$

necessity, $\alpha_{i}, \beta_{i}>0, i=1, \ldots, K$, are some constants and, as in Example $1, I_{[1]} \geq I_{[21]} \geq \ldots \geq I_{[K 1]}$. It is not difficult to check that conditions (1) and (2) of part (i) of Theorem 1 are satisfied if and only if, for $r \geq s$,

$$
\begin{aligned}
& \frac{\alpha_{r} \beta_{r}}{\left(I_{[r]}+\beta_{r} p\right)^{2}} \leq \frac{\alpha_{s} \beta_{s}}{\left(I_{[s]}+\beta_{s} p\right)}(3) \\
& \frac{\alpha_{r} \beta_{r}\left(I_{[r]}-\beta_{r} p\right)}{\left(I_{[r]}+\beta_{r} p\right)^{3}} \leq \frac{\alpha_{s} \beta_{s}\left(I_{[s]}-\beta_{s} p\right)}{\left(I_{[s]}+\beta_{s} p\right)^{3}}(4)
\end{aligned}
$$

Let $r \geq s$. Assume that the vector $\boldsymbol{I}=(0,0, \ldots, 0)$ belongs to the domain of definition of $\Phi_{m}(p, \boldsymbol{I})$. Suppose that conditions (1) and (2) of Theorem 1 are satisfied. Then from inequalities (3) and (4) for $I=(0,0, \ldots, 0)$ it follows that $\alpha_{r} / \alpha_{s}=\beta_{r} / \beta_{s}(5)$

It is easy to see that condition (3) is thus equivalent to $\beta_{r} /\left(I_{[r]}+\beta_{r} p\right) \leq \beta_{s} /\left(I_{[s]}+\beta_{s} p\right)$ or $I_{[r]} / \beta_{r} \geq I_{[s]}+\beta_{s} / \beta_{s}$. Since $I_{[r]} \leq I_{[s]}$ we conclude that, for conditions (3) and (4) to be satisfied it is necessary that (5) holds for all $r \geq s$ and, in addition, for all $r \geq s$,

$\beta_{r} \leq \beta_{s}, \alpha_{r} \leq \alpha_{s}(6)$

Suppose that the satiation level for good $\mathrm{m}$ is the same for all the consumers, that is, for $p=0$ and all $\mathrm{r}, \mathrm{s}$, $\phi\left(0, \alpha_{r}, \beta_{r}, I_{r}\right)=\phi\left(0, \alpha_{s}, \beta_{s}, I_{s}\right)$. Then from the definition of the individual demand functions $\phi$ and (5) it follows that $\alpha_{r}=\alpha_{s}$ and $\beta_{r}=\beta_{s}$ for all $\mathrm{r}$, s. Since, as is easy to see, from the above analysis it follows that inequalities (6) are strict for I[r] < I[s] if conditions (1) and (2) are satisfied, we conclude that part (i) of Theorem 1 cannot hold.

As above, we get that part (ii) of Theorem 1 holds if and only if (3) and (4) are satisfied with the inequality sign $\leq$ replaced by $\geq$. For $I[r]=I[s]=0$ this implies conditions (5). Assuming that the satiation level for good $m$ is the same for all the consumers, we get that, as above, $\alpha_{r}=\alpha_{s}$ and $\beta_{r}=\beta_{s}$ for all $\mathrm{r}$, $\mathrm{s}$. Thus, it is easy to see that part (i) of Theorem 1 holds if and only if, for all $r \geq s$,

$$
\left(I_{[r]}-\beta p\right) /\left(I_{[r]}+\beta p\right)^{3} \geq\left(I_{[s]}-\beta p\right) /\left(I_{[s]}+\beta p\right)^{3}(7)
$$

where $\beta=\beta_{r}=\beta_{s}$. Similar to Example 1 in [3], it is not difficult to check that conditions (7) are satisfied if $\left(I_{[K]} \geq\right.$ $2 \beta p$ ), that is if the income levels of all the consumers are not less than $2 \beta p$.

\section{References}

Atkinson A. B. (1970). On the measurement of inequality. Journal of Economic Theory 5, 244-263.

Dasgupta P. A., Sen A. and Starrett D. (1973). Notes on the measurement of inequality. Journal of Economic Theory 6, $180-187$. Ibragimov M. and Ibragimov R. (2007). Market demand elasticity and income inequality. Economic Theory 32, 579-587.

Lambert P. J. and Pf'ahler W. (1997). Market demand and income distribution: A theoretical exploration. Bulletin of Economic Research 49, 137-151.

Lorenz M. O. (1905). Methods of measuring concentration of wealth. Journal of the American Statistical Association 9, 209-219.

Marshall A. W. and Olkin I. (1979). Inequalities: Theory of Majorization and Its Applications. Academic Press, New York.

Saposnik R. (1993). A note on majorization theory and the evaluation of income distributions. Economics Letters 42, 179-183.

Shorrocks A. F. (1983). Ranking income distributions. Economica 50, 3-17.

Ajupov A.A., Kazakovtseva M.V. Management of Financial Stability of the Non-Tax Income of Regional Budgets // Procedia - Social and Behavioral Sciences. Volume 131, 15 May 2014, Pages 187-192/

Bagautdinova N.G., Safiullin L.N., Mubarakov I.A. The Role of Quality of Goods in Formation of Consumer Choice// Mediterranean Journal of Social Sciences.- Vol.5, No12, (2014)-pp.63-68.

Safiullin N.Z., Safiullin L.N., Gafurov I.R., Bagautdinova N.G. Distribution and product selection under uncertainty / Recent Trends in Social and Behaviour Sciences - Proceedings of the 2nd International Congress on Interdisciplinary Behavior and Social Sciences 2013, ICIBSoS 2013 PP. $309-313$.

Gallyamova D. Kh., Toumashev A.R, Malaev V.V. Influence of globalization on development of the Russian economy. Mediterranean Journal of Social Sciences.- Vol.5, No18, (2014)-pp. 133 - 137. 